\title{
Characteristics of pore migration controlled by diffusion through the pore-filling fluid
}

\author{
E. Petrishcheva $\cdot$ J. Renner
}

Received: 26 October 2009/ Accepted: 27 January 2010/Published online: 20 February 2010

(C) The Author(s) 2010. This article is published with open access at Springerlink.com

\begin{abstract}
We analyze drag and drop of pores filled with a fluid phase, e.g., water or melt, in which the constituting elements of the solid matrix are dissolved. Assuming that the diffusion through the fluid-phase dominates bulk transport kinetics, we address the problem of pore motion and calculate the pore mobility and the critical velocity of elongated and lenticular pores on a grain boundary for arbitrary dihedral angle. The found variations in critical velocity and mobility with dihedral angle are modest for given volume of pores with the two considered geometries. For given pore size, however, the dependence on dihedral angle accounts for several orders of magnitude in pore mobility and critical velocity.
\end{abstract}

Keywords Pore drag and drop · Diffusion .

Porous material $\cdot$ Analytical methods

\section{Introduction}

All solids have a boundary or an interface with their environment. In addition, the majority of solids (e.g., ceramics, metals, rocks) are polycrystalline aggregates, i.e., they have a class of internal imperfections, addressed as grain boundaries. Grain boundary networks in polycrystals share similarities with networks that are common in nature, such as in assemblages of soap bubbles and biological

E. Petrishcheva $\cdot$ J. Renner

Ruhr-University Bochum, 44780 Bochum, Germany

Present Address:

E. Petrishcheva $(\square)$

Institute of Geological Sciences, FU Berlin,

12249 Berlin, Germany

e-mail: epetris@zedat.fu-berlin.de cells, and in maps of geographical and ecological territories (Weaire and Rivier 1984). At elevated temperatures, grain boundaries in polycrystalline solids are not stationary but migrate due to various driving forces, for example reduction in total grain boundary energy (normal grain growth) (Yan et al. 1977).

Pores constitute another class of objects that is often encountered in polycrystals. A pore can be considered as a small inclusion (i.e., generally smaller than the average grain size) of a liquid or gaseous phase in a solid matrix. When the pore is empty (filled with vacancies), or occupied by a real gas, pores are often referred to as voids. Pores filled with aqueous solutions are often loosely termed fluid inclusions in geoscience and the term melt pocket is used to address pores filled with melt. The geometry of a stagnant pore depends on its position in the polycrystalline aggregate. A pore can be positioned within a grain, on the interface between two grains (grain boundary), on the line formed by the edges of three neighboring grains (triple junction), or on the point shared by four neighboring grains (four-grain junction). Pores included in a grain often exhibit approximately spherical shape indicating that the solid-fluid interfacial energy between the crystalline material and the pore-filling fluid is nearly isotropic and torques can be neglected. The shape of pores attached to grain boundaries is strongly affected by the dihedral angle

$\Phi=2 \arccos \frac{\gamma_{\mathrm{b}}}{2 \gamma_{\mathrm{s}}}$,

which in the fully isotropic case is determined by the balance of line-forces associated with grain boundary energy $\gamma_{\mathrm{b}}$ and solid-fluid (surface) interfacial energy $\gamma_{\mathrm{s}}$ at the pore tip.

The geometry of moving pores differs from that of stationary pores in the same microstructural position. 
Furthermore, two evolution scenarios are possible for pores attached to a migrating grain boundary: (1) the pore may be dragged along by the boundary or (2) it may be dropped, when the velocity of the migrating boundary is too high. The fate of pores during grain boundary migration is important for the bulk mobility of the constituents of the pore-filling fluid in the polycrystalline aggregate. Once a pore is detached from the grain boundary, its fluid content is disconnected from the grain boundary network, the most efficient pathway for transport in a polycrystal. The first evaluation of the critical (i.e., the largest possible) pore velocity $U_{\max }$ was based on Zener's ideas and resulted in the expression (Smith 1948)

$U_{\max }^{\mathrm{sph}}=\pi R_{\mathrm{p}} \gamma_{\mathrm{b}} M_{\mathrm{p}}^{\mathrm{sph}}$,

where $M_{\mathrm{p}}^{\text {sph }}$ is the mobility of a spherical pore with radius $R_{\mathrm{p}}$ attached to the boundary. Note, that the dependence on the dihedral angle and the intrinsic mechanism of pore motion are not included in this calculation, i.e., the pore has a fixed spherical shape and is uniquely characterized by a fixed mobility $M_{\mathrm{p}}^{\text {sph }}$ (see also review Nes et al. 1985 where more general yet still predetermined pore geometries are considered). In reality, pore motion may be accommodated by diffusion of matrix constituents along the solid-liquid interface, through the lattice, or through the pore-filling liquid (Hollister and Crawford 1981; Roedder 1984).

Evidently, extensions of (2) are highly desirable allowing for self-organized pore shape and mobility for both an arbitrary dihedral angle and specific transport mechanisms. Moreover, the very concept of pore mobility that underlies the derivation of (2), i.e., a linear proportionality between the applied drag force and the resulting pore velocity with a constant (velocity independent) factor $M$, may be inappropriate when separation occurs (Petrishcheva and Renner 2005). To resolve these shortcomings one has to consider the full problem of pore motion with a detailed account of the responsible transport mechanism and the resulting selforganized pore geometry. Previous work has focused on gas-filled pores for which the main mechanism for transport of matrix material is surface diffusion along the solidgas interface (see original papers Hsueh et al. 1982; Spears and Evans 1982), advanced modeling (Svoboda and Riedel 1992; Riedel and Svoboda 1993), and further improvements (Petrishcheva and Renner 2005; Huang et al. 2004). For pore motion controlled by diffusion through the porefilling fluid, only simplified estimates of mobilities and critical velocities nominally restricted to small dihedral angles can be found in the literature (Monchoux and Rabkin 2002) probably because the problem is mathematically more complicated than the corresponding surface diffusion problems. To our knowledge, a systematic investigation of the effect of dihedral angle on mobility and critical velocity of pores filled with a fluid has not been undertaken so far.

The presentation of our investigation into the behavior of pores controlled by diffusion through the pore-filling fluid is organized as follows. The foregoing "Mathematical model" is devoted to posing the mathematical problem. A simple application of the dissipation theorem is given in "Dissipation rate" in which the pore mobility is obtained for small dihedral angles. The effect of finite $\Phi$ is considered in the next two sections. After introducing a new approximate method in "Mobility and critical velocity for an elongated pore", we calculate both mobility and critical velocity for elongated pores. Lenticular pore geometry is considered in "Critical velocity and mobility for a lenticular pore", where a combination of numerical and analytical tools is used. The results are discussed and finally summarized in "Conclusions".

\section{Mathematical model}

This section is devoted to a mathematically accurate formulation of the problem at hand. It follows the strategy well-known from the related process of thermal grooving (Mullins 1960). Typically, the pore-boundary interfaces are smooth, i.e., all characteristic space scales are much larger than the distance between the atoms. Therefore, we can use well defined continuous fields for all relevant physical quantities, e.g., solution concentration or interface curvature. While it is acknowledged that the transport is realized by individual atoms, our considerations are based on a continuum approach.

We consider a pore attached to a uniformly moving grain boundary and filled with a fluid phase in which the matrix atoms are dissolved. Two different pore geometries are considered, elongated and lenticular pores (Fig. 1). The local distribution of the dissolved atoms is represented by their concentration $c(\mathbf{r}, t)$, i.e., their number per unit volume of the solution. In accord with Fick's law a spatial nonuniformity in $c$ results in a diffusive particle flux given by

$\mathbf{j}_{\text {diff }}=-D \nabla c$,
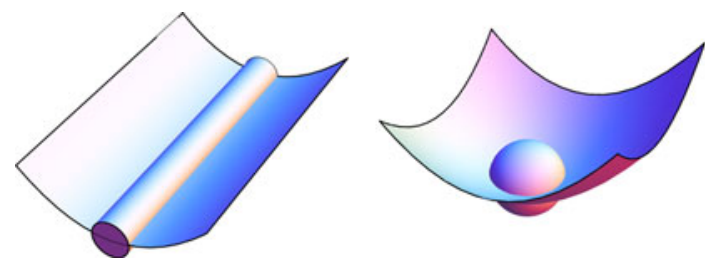

Fig. 1 Left schematic geometry of the dragged elongated pore for which the length $L_{p}$ is considerably larger then the cross-sectional dimensions. Right schematic geometry of the dragged lenticular pore 
where the diffusion coefficient $D$ corresponds to the random motion of the matrix atoms in the solution and is assumed to be independent of $c$. The total number of the dissolved matrix atoms changes only on the pore boundaries where dissolution-precipitation occurs. Therefore, a driftdiffusion particle balance equation (de Groot 1951) can be applied inside the pore

$\frac{\partial c}{\partial t}+\nabla(c \mathbf{v}-D \nabla c)=0$,

where $\mathbf{j}_{\text {mac }}=c \mathbf{v}$ is the macroscopic flux induced by the macroscopic velocity $\mathbf{v}(\mathbf{r}, t)$ of the pore fluid. Actually, we will neglect the macroscopic term in Eq. 4 in the following. Consider, e.g., a lenticular pore with the radius $R_{\mathrm{p}}$ attached to the boundary moving upwards with the velocity $U$ (Fig. 2). The total number of matrix atoms that diffuse across the pore per second reads $J_{\text {diff }}=\pi R_{\mathrm{p}}^{2} U / \Omega$, where $\Omega$ is the atomic volume of the constituents of the matrix. Comparing $J_{\text {diff }}$ with $J_{\mathrm{mac}}=\pi R_{\mathrm{p}}^{2} \cdot c U$ we see that the macroscopic transport can be neglected if $c \Omega \ll 1$. This inequality is rather non-restrictive simply requiring that the solute concentration remains small compared to the concentration of constituents of the solvent. Furthermore, the characteristic time of the diffusive evolution of $c$ is given by $R_{\mathrm{p}}^{2} / D$ and the characteristic time of pore motion can be estimated as $R_{\mathrm{p}} / U$. Assuming that the first time is much smaller than the second, i.e., assuming that the pore velocity is not too large $U \ll D / R_{\mathrm{p}}$, we conclude that the equilibrium concentration of $c$ is quickly established and is finally determined by the Laplace equation

$\nabla^{2} c=0$.

Using the modern terminology of synergetics (Haken 2004), one can say that the concentration $c$ is "slaved". It is important to note that $c$ still varies with time. The variation is

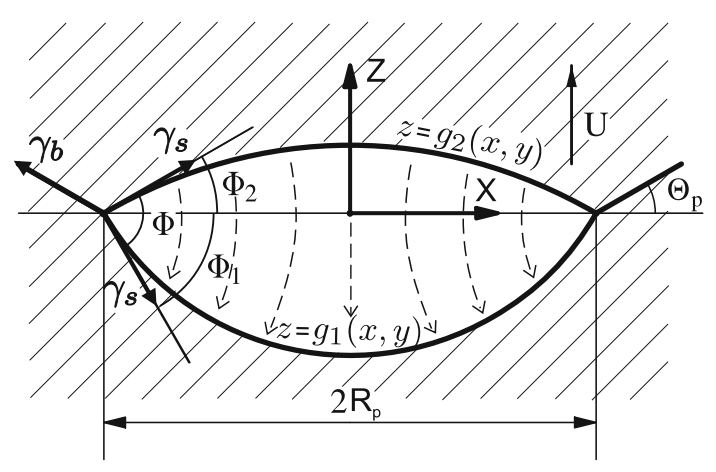

Fig. 2 Pore motion realized by diffusion through the pore-filling fluid. The curvature of the leading surface is smaller than that of the trailing surface. Therefore, the concentration at the leading surface is larger than that at the trailing surface in accord with (8). The concentration gradient results in the diffusion flux shown by the dashed arrows. This downwards transfer of matter is responsible for the upwards motion of the pore slow in the sense that the time derivative in (4) can be ignored. The time dependence is not directly determined by diffusion through the pore-filling fluid but by an additional process. In our case, the additional process is the slow motion of the boundary to which the pore is attached. This process is addressed by time dependent boundary conditions for (5). In particular, (5) has to be solved in a moving region

$g_{1}(x, y, t)<z<g_{2}(x, y, t)$

where $g_{1,2}(x, y, t)$ represent the trailing and leading pore surfaces, respectively. These surfaces are self-organized and intersect at the dihedral angle $\Phi$ defined by Eq. 1.

To formulate the boundary conditions for Eq. 5 on the pore surface, we now introduce an equilibrium concentration $c_{0}$ at which the rates of dissolution and precipitation at a plane interface between the solid matrix and the solution are equal (at given pressure and temperature). Starting from a non-equilibrium situation at the solid-fluid interface, there is a characteristic time for the equilibrium concentration to establish. The migration processes of interest are considered much slower than this equilibration time, i.e., the equilibrium concentration $c=c_{0}$ is established quasiinstantaneously.

When the pore surface is curved, the equilibrium concentration of the solution is different from $c_{0}$ and is denoted by $c_{\mathrm{s}}$ such that the sought boundary conditions read

$\left.c(\mathbf{r}, t)\right|_{z=g_{i}(x, y, t)}=c_{\mathrm{s} i}$,

for $i=1,2$. The specific value of $c_{\mathrm{s}}$ is defined by the Gibbs-Thompson relation (Partington 1952; Balluffi et al. 2005) which for the case at hand reads (Mullins 1960)

$c_{\mathrm{s}}=c_{0}(1-\Gamma \kappa)$,

where $\kappa$ denotes the mean curvature of the interface and a constant proportionality factor $\Gamma$ reads

$\Gamma=\frac{\gamma_{s} \Omega}{k T}$

and $k T$ is temperature in energetic units. For typical parameters $\Gamma \simeq 1 \mathrm{~nm}$ (see, e.g., Monchoux and Rabkin 2002; Mullins 1960).

The curvature $\kappa$ is defined positive when the pore is convex with respect to the solid, e.g., for a spherical pore, and negative otherwise. It is given by the following expressions (see for example, Dubrovin and Fomenko 1992)

$\kappa_{1,2}= \pm \operatorname{div}\left(\frac{\nabla g_{1,2}}{\sqrt{1+\left|\nabla g_{1,2}\right|^{2}}}\right)$

for the trailing and the leading surfaces, respectively. In particular $\kappa>0$ and $c_{\mathrm{s}}<c_{0}$, for a spherical pore and more generally for a convex pore consisting of two spherical segments. When the curvatures of the leading and the 
trailing surfaces differ, a gradient of solution concentration is created inside the pore. In conjunction with dissolutionprecipitation, a diffusion flux transports material through the pore and thus enables the motion of the pore relative to the matrix (see Fig. 2).

Equations (5) and (7), that determine the concentration of the matrix atoms in the solution, do not contain a time derivative. Instead, the time derivative enters the basic equations through the general geometric identity for the moving surface $z=g_{i}(x, y, t)$, namely

$\frac{\partial g_{i}}{\partial t}=v_{\mathrm{n}} \sqrt{1+\left|\nabla g_{i}\right|^{2}}$,

where $i=1,2$ and $v_{\mathrm{n}}$ denotes the normal component of the surface velocity. For a complete closed mathematical model, we finally need a specific expression for $v_{\mathrm{n}}$ for the leading and trailing surfaces. To derive it, we consider an element $\mathrm{d} S$ of the pore surface. For a normal displacement of $v_{\mathrm{n}} \mathrm{d} t$, the corresponding increase in grain volume is $\mathrm{d} S v_{\mathrm{n}} \mathrm{d} t$ and the ratio $\mathrm{d} S v_{\mathrm{n}} \mathrm{d} t / \Omega$ gives the net gain (precipitation minus dissolution) of matrix atoms. These atoms are supplied by the transport through the pore-filling fluid. For slow pore motion and under the condition $c \Omega \ll 1$, the diffusive transport dominates and the net gain equals $-\left(\mathbf{j}_{\text {diff }}\right)_{n} \mathrm{~d} S \mathrm{~d} t$, where the flux is projected on the outward (with respect to the grain) normal vector $\mathbf{n}$ and is negative for positive $v_{\mathrm{n}}$. Therefore,

$\left(\mathbf{j}_{\text {diff }}\right)_{\mathrm{n}}=-\frac{v_{\mathrm{n}}}{\Omega}$.

Using Fick's law (3) we obtain

$v_{\mathrm{n}}=D \Omega(\nabla c)_{\mathrm{n}}=\left.D \Omega(\mathbf{n} \cdot \nabla c)\right|_{z=g_{i}(x, y, t)}$

for $i=1,2$. Equation (13) provides the necessary expressions for the velocities of interfaces and gives the purely geometrical Eq. 11 a physical meaning.

The system (5-13) can be used as follows. For a given shape (6) of trailing and leading surfaces at time $t$, the boundary values $c_{\mathrm{s}}$ are calculated in accord with (8). Then, the stationary Eq. 5 is solved inside the pore region (6) with the boundary conditions (7). The calculated concentration $c$ is used to obtain boundary velocities $v_{\mathrm{n} 1}$ and $v_{\mathrm{n} 2}$ in accord with (13) that are further used in (11) to obtain the new shape of the pore surfaces preserving the value of $\Phi$ at time $t+\mathrm{d} t$ and so on. In practice, this scheme may have to be simplified using different approximations that we discuss in the following.

\section{Dissipation rate}

Pore motion inside the matrix is accompanied by a permanent decrease of the free energy. The corresponding dissipation rate $\Delta_{\mathrm{p}}$ is related with the macroscopical flux $\mathbf{j}$ inside the pore and can be presented as (Prigogine 1967)

$\Delta_{\mathrm{p}}=\frac{k T}{D c_{0}} \iiint \mathbf{j}^{2} \mathrm{~d} x \mathrm{~d} y \mathrm{~d} z$,

where the integration is performed over the pore volume. Equation 12 indicates that the particle flux $\mathbf{j}$ inside the pore moving with the velocity $U$ can be estimated as $U / \Omega$ at least for small dihedral angles. Using this estimate we obtain that

$\Delta_{\mathrm{p}} \approx \frac{k T}{D c_{0}} \frac{U^{2}}{\Omega^{2}} V_{\mathrm{p}}$,

where $V_{\mathrm{p}}$ is the pore volume. On other hand the dissipation rate is determined by the drag force $K_{\text {drag }}$ and the resulting pore velocity $U$ as

$\Delta_{\mathrm{p}}=K_{\mathrm{drag}} U$.

Combining the latter two expressions we obtain a simple estimate for the pore mobility (Monchoux and Rabkin 2002)

$M_{\mathrm{p}}=\frac{U}{K_{\mathrm{drag}}} \approx \frac{D \Omega^{2} c_{0}}{V_{\mathrm{p}} k T}$.

Here $V_{\mathrm{p}}$ is associated with the equilibrium pore volume. In the following two different pore geometries will be considered (Fig. 1). First we will consider an elongated pore, the corresponding "large" space scale is denoted by $L_{\mathrm{p}}$. A cross-section of the equilibrium pore is similar to that shown in Fig. 2; however, with identical curvature radius $R_{\text {eq }}$ of the two pore segments, namely

$R_{\mathrm{eq}}=\frac{R_{\mathrm{p}}}{\sin (\Phi / 2)}$.

The volume of an equilibrium pore is given by

$V_{\mathrm{p}}^{2 \mathrm{D}}=\frac{\Phi-\sin (\Phi)}{\sin ^{2}(\Phi / 2)} R_{\mathrm{p}}^{2} L_{\mathrm{p}}$.

The second geometry corresponds to a lenticular pore for which the volume is given by

$V_{\mathrm{p}}^{3 \mathrm{D}}=\frac{4}{3} \pi R_{\mathrm{p}}^{3} \frac{1-3 / 2 \cos (\Phi / 2)+1 / 2 \cos ^{3}(\Phi / 2)}{\sin ^{3}(\Phi / 2)}$.

We recall that Eq. 14 is an approximation which becomes exact for $\Phi \rightarrow 0$. Pores with arbitrary dihedral angles are considered in the next two sections.

\section{Mobility and critical velocity for an elongated pore}

In this section, we apply a simple analytical method to find both mobility and critical velocity of an elongated, channel-like pore for an arbitrary dihedral angle. Note, the 
following two-dimensional analysis equally applies to elongated and quasi two-dimensional pores in thin films (Thompson and Carel 1996). Lenticular pores are considered in the next section where analytical and numerical approaches are combined.

Let us consider an elongated pore parallel to the $O Y$ axis. Along the $O X$ axis the pore is positioned in the region $-R_{\mathrm{p}}<x<R_{\mathrm{p}}$; the pore tips are at $x= \pm R_{\mathrm{p}}$. We assume that the two branches of either a stationary or a moving pore always meet at a fixed dihedral angle $\Phi$ (see Fig. 2). The equilibrium pore shape is given by two identical circular segments. The constant solution concentration inside such an equilibrium pore results from (8) to

$c_{\text {eq }}=c_{0}\left(1-\frac{\Gamma}{R_{\text {eq }}}\right)$

considering that one of the two principal curvatures of any channel-like pore is zero. Here $R_{\text {eq }}$ is given by Eq. 15 .

We now consider a pore that moves upwards with a constant velocity $U$. The motion is induced by the motion of a boundary between two grains of the solid matrix. The shape of the moving pore is approximated by two circular segments that in contrast to the equilibrium shape at rest exhibit different radii of curvature. Approximating the pore shape by circular segments is reasonable. Only in the immediate vicinity of the pore tips, the continuity of the solution concentration requires continuity of the curvature and therefore a deviation from the circle geometry.

The radii of the leading and trailing surfaces self-organize in response to the pore motion and their different values are denoted by $R_{2}$ and $R_{1}$, respectively. In accord with (8), the boundary values of the solution concentration are

$c_{\mathrm{s} 2}=c_{0}\left(1-\frac{\Gamma}{R_{2}}\right) \quad$ and $\quad c_{\mathrm{s} 1}=c_{0}\left(1-\frac{\Gamma}{R_{1}}\right)$,

where $R_{2}>R_{1}$ and therefore $c_{0}>c_{\mathrm{s} 2}>c_{\mathrm{s} 1}$. Note, that for small velocities the pore is convex with respect to the matrix and the corresponding curvatures are positive. However, the leading surface can become concave with increasing velocity (Fig. 4). In this case, the corresponding curvature becomes negative and the solution concentration at the leading surface reads $c_{\mathrm{s} 2}=c_{0}\left(1+\Gamma / R_{2}\right)$ and $c_{\mathrm{s} 2}>c_{0}>c_{\mathrm{s} 1}$. In both situations, the concentration at the leading surface $c_{\mathrm{s} 2}$ is larger than that at the trailing surface $c_{\mathrm{s} 1}$. Therefore, a diffusive transport directed downwards is induced inside the pore, that causes the upwards motion of the pore.

The solution concentration $c(x, z)$ inside the pore can be found from the Laplace equation (5) in two dimensions $\frac{\partial^{2} c}{\partial x^{2}}+\frac{\partial^{2} c}{\partial z^{2}}=0$

with the boundary conditions

$\left.c\right|_{\text {leading }}=c_{0}\left[1-\frac{\Gamma}{R_{\mathrm{p}}} \sin \left(\Phi_{2}\right)\right]$,

$\left.c\right|_{\text {trailing }}=c_{0}\left[1-\frac{\Gamma}{R_{\mathrm{p}}} \sin \left(\Phi_{1}\right)\right]$,

where $\Phi_{1,2}$ denote the angles between the pore branches and the $O X$ axis (Fig. 2) related to the dihedral angle $\Phi=\Phi_{1}+\Phi_{2}$ and the pore drag angle $\Theta_{\mathrm{p}}$ according to

$\Phi_{1,2}=\frac{1}{2} \Phi \pm \Theta_{\mathrm{p}}$.

By considering leading and trailing surfaces as perfect cylindrical (or circular) segments with different radii we make an approximation that bears limitations. Improvements are particularly warranted near the pore tips where the approximation and the resulting values of $c_{\mathrm{s} 1} \neq c_{\mathrm{s} 2}$ are in conflict with the continuity of $c(\mathbf{r}, t)$ inside the pore. The advantage is that the mathematical problem, as was initially given in "Mathematical model" is greatly simplified. In fact, the simplified two-dimensional problem has an exact solution. The key point is that a test function (Lavrentjev and Shabat 1998)

$c_{\text {test }}(x, z) \sim \arctan \left(\frac{2 z}{1-x^{2}-z^{2}}\right)$

satisfies the Laplace equation (20) and exhibits identical values at each circle segment connecting two points at $x= \pm 1$. One can directly check that these values are $-\Phi_{1}$ and $\Phi_{2}$ for the segments shown in Fig. 2. We can therefore look for a solution of our problem in the form

$\frac{c}{c_{0}}=A \arctan \left(\frac{2 R_{\mathrm{p}} z}{R_{\mathrm{p}}^{2}-x^{2}-z^{2}}\right)+B$,

where $A$ and $B$ are suitable constants and we scaled the coordinates to yield pore tips at $x= \pm R_{\mathrm{p}}$. To obtain $A$ and $B$ one solves the following system

$$
\begin{gathered}
-A \Phi_{1}+B=1-\frac{\Gamma}{R_{\mathrm{p}}} \sin \left(\Phi_{1}\right), \\
A \Phi_{2}+B=1-\frac{\Gamma}{R_{\mathrm{p}}} \sin \left(\Phi_{2}\right),
\end{gathered}
$$

which results from the boundary conditions. Actually, only $A$ is of interest because $B$ does not contribute to the particle flux $\mathbf{j} \propto \nabla c$ see (24). Using (23) we finally find that

$A=\frac{2 \Gamma \cos (\Phi / 2)}{R_{\mathrm{p}} \Phi} \sin \left(\Theta_{\mathrm{p}}\right)$. 
Equations 24 and 25 uniquely determine the diffusive flux in the moving pore for given values of the parameters $R_{\mathrm{p}}$, $\Gamma, \Phi$, and $\Theta_{\mathrm{p}}$.

We now calculate the pore velocity from $\left.j_{z}\right|_{z=0}$, which amounts to (3)

$\left.j_{z}\right|_{z=0}=-\left.D \frac{\partial c}{\partial z}\right|_{z=0}=-\frac{4 \cos (\Phi / 2)}{\Phi} \frac{D \Gamma c_{0}}{R_{\mathrm{p}}^{2}-x^{2}} \sin \left(\Theta_{\mathrm{p}}\right)$.

The total flux (i.e., the total number of atoms transferred per second), $J$, follows upon integration over the pore cross-section in the XOY plane,

$J=\iint\left(-\left.j_{z}\right|_{z=0}\right) \mathrm{d} x \mathrm{~d} y=L_{\mathrm{p}} \int_{-R_{\mathrm{p}}}^{R_{\mathrm{p}}}\left(-\left.j_{z}\right|_{z=0}\right) \mathrm{d} x$,

and is related to the pore velocity by

$J \Omega=2 R_{\mathrm{p}} L_{\mathrm{p}} U^{2 \mathrm{D}}$.

Therefore, the pore velocity follows as

$U^{2 \mathrm{D}}=\frac{2 \cos (\Phi / 2)}{\Phi} \frac{D \Omega \Gamma c_{0}}{R_{\mathrm{p}}} \sin \left(\Theta_{\mathrm{p}}\right) \int_{-R_{\mathrm{p}}}^{R_{\mathrm{p}}} \frac{\mathrm{d} x}{R_{\mathrm{p}}^{2}-x^{2}}$.

At first glance, the last expression is problematic because the integral diverges. However, the singularity of the total flux $J$ is an artifact of approximating the geometry of the moving pore by two circle segments. The gradient of concentration diverges when approaching the pore tips formed by two different circle segments in accord with (8), consequently the local particle flux (3) diverges too. In reality, however, the geometry of the problem suggests that the flux decreases towards the pore tips and that the contribution of the flux in the vicinity of the pore tips has to remain finite. In the following, we restrict integration to the region $|x|<R_{\mathrm{p}}-\delta$

$\int_{-R_{\mathrm{p}}+\delta}^{R_{\mathrm{p}}-\delta} \frac{\mathrm{d} x}{R_{\mathrm{p}}^{2}-x^{2}}=\frac{1}{R_{\mathrm{p}}} \ln \frac{2 R_{\mathrm{p}}-\delta}{\delta}$

yielding a finite expression

$U^{2 \mathrm{D}}=\frac{2 \cos (\Phi / 2)}{\Phi} \frac{D \Omega \Gamma c_{0}}{R_{\mathrm{p}}^{2}} \sin \left(\Theta_{\mathrm{p}}\right) \ln \frac{2 R_{\mathrm{p}}-\delta}{\delta}$

in which a specific value of $\delta$ will be quantified below.

In general, our use of a regularization parameter follows a well known strategy for diverging Landau's integral (Landau and Lifshitz 1981). Three considerations support that restricting the integral limits yields robust and physically meaningful results. First, the divergence of the flux is moderate and the result of the restricted integration is insensitive to the exact choice of $\delta / R_{\mathrm{p}}$ due to the logarithmic dependence. Second, the quantity $\Gamma$ constitutes a finite physical lower bound for $\delta$ because it quantifies the characteristic length scale of possible changes in solution concentration and interface curvature (8). The logarithmic factor is then less than $\ln \left(2 R_{\mathrm{p}} / \Gamma\right)$ so that the divergence is actually avoided. Third, the intuitive notion of a subordinate flux in the vicinity of the pore tips is shown to hold for the motion of a pore close to equilibrium geometry (see "Appendix"). From this explicit analysis, we find that the relative contribution of the tip region to the total flux is only of order $\delta / R_{\mathrm{p}}$ independent of dihedral angle.

In the following, we chose $\delta$ in such a way that the result (14) of the previous section for the pore mobility is exactly reproduced for small dihedral angles (see Eq. 31 of the next section). This requires

$\ln \frac{2 R_{\mathrm{p}}-\delta}{\delta}=3$ and $\delta=0.095 R_{\mathrm{p}}$

in agreement with our assumption that $\delta \ll R_{\mathrm{p}}$. Equation 26 becomes

$U^{2 \mathrm{D}}=\frac{6 \cos (\Phi / 2)}{\Phi} \frac{D \Omega \Gamma c_{0}}{R_{\mathrm{p}}^{2}} \sin \left(\Theta_{\mathrm{p}}\right)$.

Finally, we recall that $\cos (\Phi / 2)$ is proportional to $\gamma_{b} / \gamma_{s}(1)$ and that $\Gamma$ is proportional to $\gamma_{\mathrm{s}}(9)$. Thus, the resulting expression for the pore velocity reads

$U^{2 \mathrm{D}}=\frac{3}{\Phi} \frac{\gamma_{\mathrm{b}} D \Omega^{2} c_{0}}{R_{\mathrm{p}}^{2} k T} \sin \left(\Theta_{\mathrm{p}}\right)$,

an expression that we now use to quantify pore mobility and critical velocity.

Mobility of an elongated pore

In this section, we assume that the pore velocity is so small that the distortion of the pore shape relative to the equilibrium shape is negligible and the pore volume is still determined by Eq. 16. The drag force amounts to

$K_{\text {drag }}=2 L_{\mathrm{p}} \gamma_{\mathrm{b}} \sin \left(\Theta_{\mathrm{p}}\right)$

and therefore (29) gives a pore mobility of

$M_{\mathrm{p}}^{2 \mathrm{D}}=\frac{U^{2 \mathrm{D}}}{K_{\mathrm{drag}}}=\frac{3}{2 \Phi} \frac{D \Omega^{2} c_{0}}{L_{\mathrm{p}} R_{\mathrm{p}}^{2} \mathrm{kT}}$.

It is useful to rewrite the last equation in terms of pore volume. Using Eq. 16 we arrive at

$M_{\mathrm{p}}^{2 \mathrm{D}}=\frac{3}{2} \frac{\Phi-\sin (\Phi)}{\Phi \sin ^{2}(\Phi / 2)} \frac{D \Omega^{2} c_{0}}{V_{\mathrm{p}}^{2 \mathrm{D}} k T}$.

The latter expression is finite for all values of $\Phi \in[0, \pi]$ and is our main result for the mobility of elongated (twodimensional) pores (Fig. 3). In particular, 


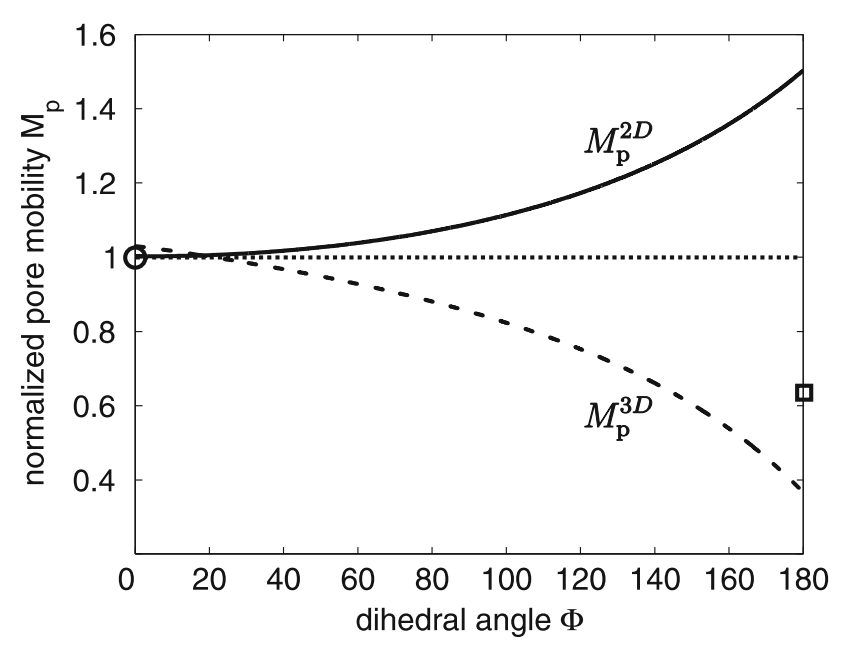

Fig. 3 Pore mobilities $M_{\mathrm{p}}^{2 \mathrm{D}}$ (solid line) and $M_{\mathrm{p}}^{3 \mathrm{D}}$ (dashed line) normalized by $D \Omega^{2} c_{0} /\left(V_{\mathrm{p}} k T\right)$ versus dihedral angle $\Phi$ in accord with Eqs. 31 and 40. Approximate results of (Monchoux and Rabkin 2002), which are identical for both elongated and lenticular pores are shown by the dotted line. The open circle represents the exact result for $\Phi \rightarrow 0$. The open square represents the classical prediction for a spherical pore (41)

$\left.M_{\mathrm{p}}^{2 \mathrm{D}}\right|_{\Phi \rightarrow 0}=\frac{D \Omega^{2} c_{0}}{V_{\mathrm{p}}^{2 \mathrm{D}} k T} \quad$ and $\left.\quad M_{\mathrm{p}}^{2 \mathrm{D}}\right|_{\Phi \rightarrow \pi}=1.5 \frac{D \Omega^{2} c_{0}}{V_{\mathrm{p}}^{2 \mathrm{D}} k T}$.

We conclude that pore mobility depends only slightly on dihedral angle for a given pore volume.

Critical velocity of an elongated pore

The maximal pore velocity associated with Eq. 29 reads

$U_{\max }^{2 \mathrm{D}}=\frac{3}{\Phi} \frac{\gamma_{\mathrm{b}} D \Omega^{2} c_{0}}{R_{\mathrm{p}}^{2} k T}$

our main result for the separation problem in the case that diffusion through the pore-filling fluid controls the motion of an elongated pore. Note, the critical velocity of pores with fixed size $R_{\mathrm{p}}$ becomes large when $\Phi$ is small. Indeed, when the leading surface becomes concave down (as in last row in Fig. 4) and $\Phi \rightarrow 0$, the finite jump between $c_{\mathrm{s} 1}$ and $c_{\mathrm{s} 2}$ occurs on a small distance of order $R_{\mathrm{p}} \Phi$ causing a large diffusion flux (Raj 1982). Correspondingly, the resulting pore velocity is also large in accord with (32). When, instead of pores of given $R_{\mathrm{p}}$, one compares pores with given volume but different dihedral angles, $R_{\mathrm{p}}$ increases with the decrease of $\Phi$. For a channel-like pore of given volume, $R_{\mathrm{p}}^{2} \Phi \simeq$ const so that the critical velocity remains limited also for small dihedral angles.

\section{Critical velocity and mobility for a lenticular pore}

With small modifications, the strategy followed for elongated pores in the previous section can be applied to lenticular pores, too. The pore-boundary interfaces are approximated by spherical caps (Fig. 2, where the pore cross-section has now rotational symmetry around the $O Z$ axis). The boundary conditions read

$c_{\mathrm{s} 2}=c_{0}\left(1-\frac{2 \Gamma}{R_{2}}\right), \quad c_{\mathrm{s} 1}=c_{0}\left(1-\frac{2 \Gamma}{R_{1}}\right)$.

Equation (20) is replaced by the radially symmetric version of (5), i.e.,

$\frac{1}{\rho} \frac{\partial}{\partial \rho}\left(\rho \frac{\partial c}{\partial \rho}\right)+\frac{\partial^{2} c}{\partial z^{2}}=0$,

where $\rho=\sqrt{x^{2}+y^{2}}$ and the pore is located in the region $\rho<R_{\mathrm{p}}$.

The solution strategy is as follows. All lengths are normalized by $R_{\mathrm{p}}$. We first fix the dihedral angle $\Phi$ and choose several values for the drag angle $\Theta_{p}$ for which the geometry of the pore, in particular the radii of the spherical segments $R_{1,2}=R_{\mathrm{p}} / \sin \left(\Phi_{1,2}\right)$, follow according to (23). The Laplace equation (34) is then solved numerically within the pore for the space distribution of $c(\rho, z)$. In fact, only the difference $c-c_{0}$ has to be calculated, the latter is normalized according to

$\bar{c}=-\frac{R_{\mathrm{p}}}{\Gamma}\left(\frac{c}{c_{0}}-1\right)$

as suggested by (24). The negative sign ensures positive value of $\bar{c}$. The normalized boundary conditions read

$\bar{c}_{\mathrm{s} 2}=2 \sin \left(\Phi_{2}\right) \quad$ and $\quad \bar{c}_{\mathrm{s} 1}=2 \sin \left(\Phi_{1}\right)$.

Examples of numerical solutions are shown in Fig. 4. For each specific solution, one can calculate the particle flux (3)

$\mathbf{j}=\frac{D \Gamma c_{0}}{R_{\mathrm{p}}^{2}} \bar{\nabla} \bar{c}$

where $\bar{\nabla}$ refers to the normalized coordinates. The flux is integrated to get the total number of atoms that are transferred across the pore per second

$J=\frac{D \Gamma c_{0}}{R_{\mathrm{p}}^{2}} I$

where the integral $I$ is determined over the trailing pore surface

$I=\iint(\mathbf{n} \bar{\nabla} \bar{c}) \mathrm{d} S=R_{\mathrm{p}}^{2} \iint(\mathbf{n} \bar{\nabla} \bar{c}) \mathrm{d} \bar{x} \mathrm{~d} \bar{y}$

and $\mathbf{n}$ denotes the corresponding unit normal vector. Similar to the approach in the previous section the integration is actually performed excluding the vicinity of the pore tip. Choosing $\bar{\rho}<0.93$ the predictions of "Dissipation rate" for small dihedral angles are reproduced to a good accuracy. 
Fig. 4 Density plots of normalized concentration $\bar{c}(\bar{\rho}, \bar{z})$ in a lenticular pore obtained solving (34) numerically with the boundary conditions (33) for a dihedral angle of $\Phi=\pi / 3$ and indicated values of the drag angle $\Theta_{p}$
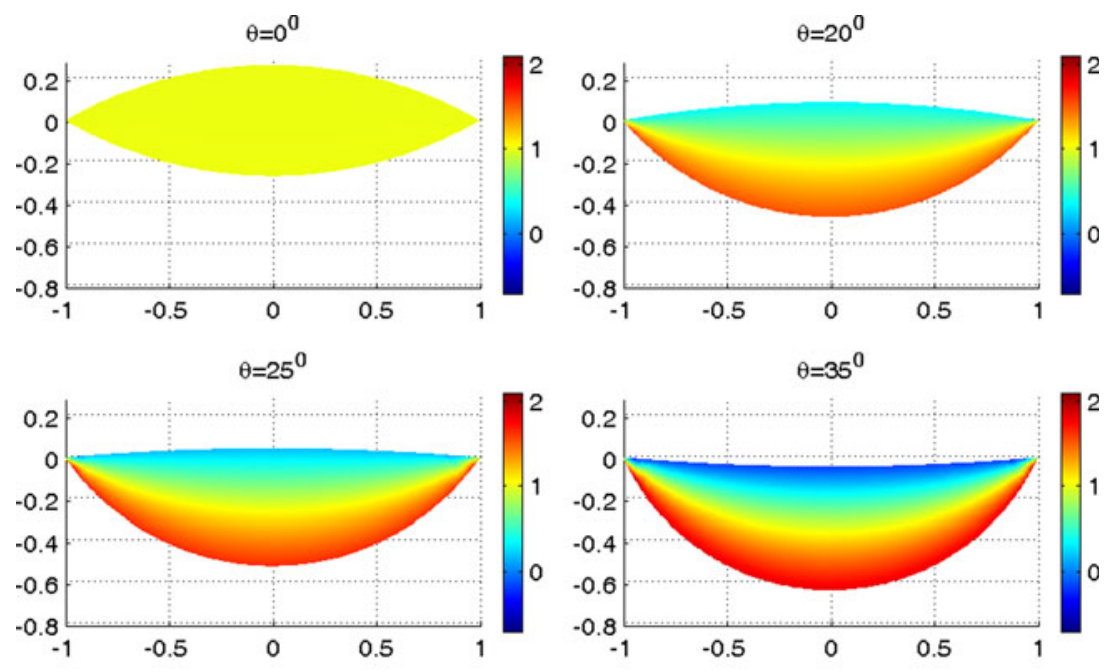

$\theta=40^{0}$
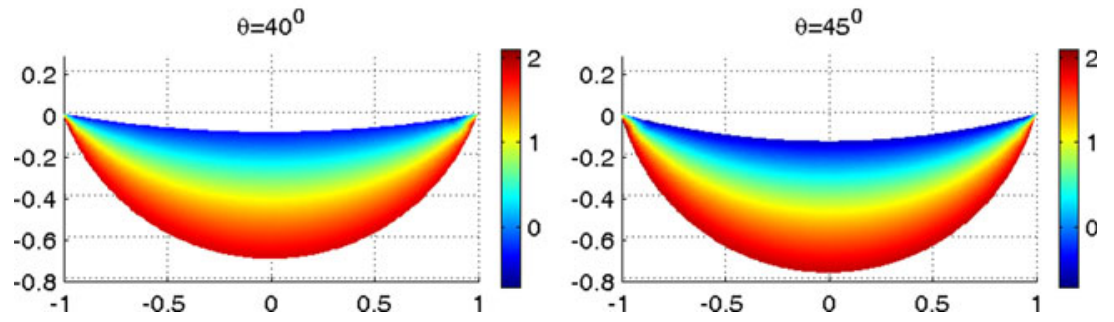

The restriction of the integration limits is justified as in the case of elongated pores. In particular, analyzing the motion of a pore close to equilibrium geometry demonstrates that the relative contribution of the excluded region to the total flux is only on the order of $2 \delta / R_{\mathrm{p}}$ (see "Appendix") again independent of dihedral angle. The velocity of a lenticular pore is calculates as

$U^{3 \mathrm{D}}=\frac{J \Omega}{\pi R_{\mathrm{p}}^{2}}=\frac{D \Omega \Gamma c_{0}}{R_{\mathrm{p}}^{2}} \frac{1}{\pi} \iint(\mathbf{n} \bar{\nabla} \bar{c}) \mathrm{d} \bar{x} \mathrm{~d} \bar{y}$.

Velocity normalized in accord with (35)

$u^{3 \mathrm{D}}=U^{3 \mathrm{D}} / \frac{D \Omega \Gamma c_{0}}{R_{\mathrm{p}}^{2}}$

exhibits a maximum at some drag angle for any given value of dihedral angle (Fig. 5). The maximum is, however, not reached for $\Theta_{p}=\pi / 2$ as for elongated pores (28), but for intermediate values of the drag angle that systematically decrease with increasing dihedral angle.

The numerical results for $u_{\max }^{3 \mathrm{D}}(\Phi)$ can be closely approximated by an analytical expression in analogy to Eq. 28

$u_{\max }^{3 \mathrm{D}}=C_{\mathrm{u}}^{3 \mathrm{D}}(\Phi) \frac{\cos (\Phi / 2)}{\Phi}$,

where numerical fitting yields

$C_{\mathrm{u}}^{3 \mathrm{D}}(\Phi)=11.48-5.54 \Phi+0.67 \Phi^{2}$.

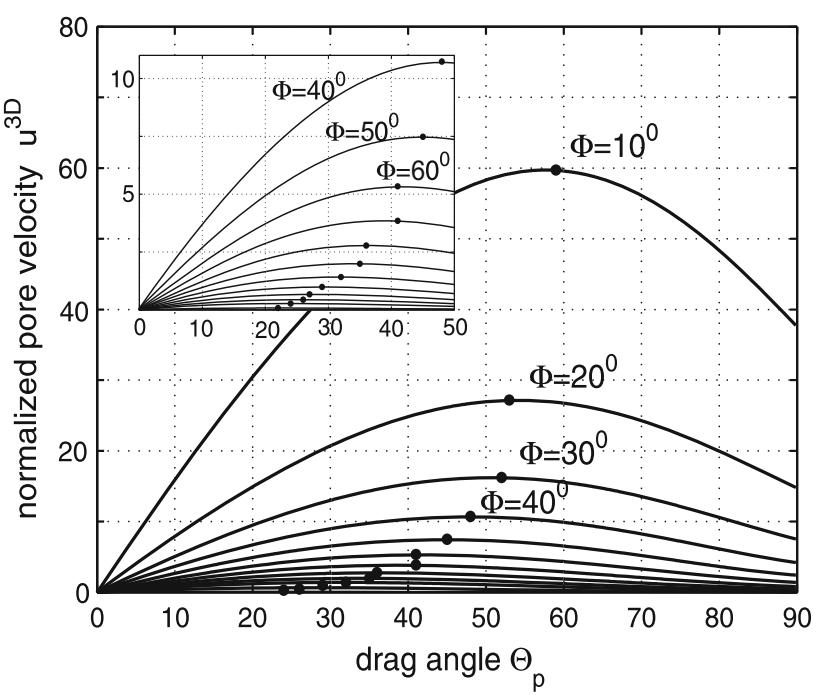

Fig. 5 Pore velocity normalized by $D \Omega \Gamma c_{0} / R_{\mathrm{p}}^{2}$ for a lenticular pore versus drag angle $\Theta_{\mathrm{p}}$ for indicated values of the dihedral angle $\Phi$. The critical pore velocities are highlighted by points. For large dihedral angles, the relations are magnified in the inset

Using Eq. 36 and returning to physical units, we obtain

$U_{\max }^{3 \mathrm{D}}=\frac{C_{\mathrm{u}}^{3 \mathrm{D}}(\Phi)}{2 \Phi} \frac{\gamma_{\mathrm{b}} D \Omega^{2} c_{0}}{R_{\mathrm{p}}^{2} k T}$.

Notably, $U_{\max }^{3 \mathrm{D}}$ remains finite for $\Phi \rightarrow \pi$. Equation 38 is our main result regarding the separation problem for a lenticular pore. 
The mobility of a lenticular pore can be estimated from the ratio between the pore velocity and the drag force constrained by the numerical results presented in Fig. 5 . We consider only small drag angles, thus the drag force

$K_{\text {drag }}=2 \pi R_{\mathrm{p}} \gamma_{\mathrm{b}} \sin \left(\Theta_{\mathrm{p}}\right) \approx 2 \pi R_{\mathrm{p}} \gamma_{\mathrm{b}} \Theta_{\mathrm{p}}$.

Pore mobility is given by the expression

$M_{\mathrm{p}}^{3 \mathrm{D}}=\frac{U^{3 \mathrm{D}}}{K_{\text {drag }}}=\frac{u^{3 \mathrm{D}}}{\Theta_{\mathrm{p}}} \frac{\gamma_{\mathrm{s}} D \Omega^{2} c_{0}}{2 \pi \gamma_{\mathrm{b}} R_{\mathrm{p}}^{3} k T}$,

in which our numerical results for $u^{3 \mathrm{D}} / \Theta_{\mathrm{p}}$ from Fig. 5 can be presented by an analytical approximation similar to Eq. 36

$\frac{u^{3 \mathrm{D}}}{\Theta_{\mathrm{p}}}=C_{\mathrm{m}}^{3 \mathrm{D}}(\Phi) \frac{\cos (\Phi / 2)}{\Phi}$

with

$C_{\mathrm{m}}^{3 \mathrm{D}}(\Phi)=16.41-1.27 \Phi-0.91 \Phi^{2}$.

The result for the pore mobility reads

$M_{\mathrm{p}}^{3 \mathrm{D}}=\frac{C_{\mathrm{m}}^{3 \mathrm{D}}(\Phi)}{\Phi} \frac{D \Omega^{2} c_{0}}{4 \pi R_{\mathrm{p}}^{3} k T}$.

It can be finally expressed in the form

$M_{\mathrm{p}}^{3 \mathrm{D}}=\frac{C_{\mathrm{m}}^{3 \mathrm{D}}(\Phi)}{\Phi} \frac{2-3 \cos (\Phi / 2)+\cos ^{3}(\Phi / 2)}{6 \sin ^{3}(\Phi / 2)} \frac{D \Omega^{2} c_{0}}{V_{\mathrm{p}}^{3 \mathrm{D}} k T}$,

where $V_{\mathrm{p}}^{3 \mathrm{D}}$ denotes the pore volume given by Eq. 17. For a given pore volume Eq. 40 yields finite mobilities for all values of $\Phi \in[0, \pi]$. In particular,

$\left.M_{\mathrm{p}}^{3 \mathrm{D}}\right|_{\Phi \rightarrow 0}=1.02 \frac{D \Omega^{2} c_{0}}{V_{\mathrm{p}}^{3 \mathrm{D}} k T} \quad$ and $\left.\quad M_{\mathrm{p}}^{3 \mathrm{D}}\right|_{\Phi \rightarrow \pi}=0.36 \frac{D \Omega^{2} c_{0}}{V_{\mathrm{p}}^{3 \mathrm{D}} k T}$.

Note, that $M_{\mathrm{p}}^{3 \mathrm{D}} \mathrm{I}_{\Phi \rightarrow 0}$ reasonably agrees with the prediction (14). The mobility at given pore volume decreases about three times with increasing dihedral angle (Fig. 3).

The variations of pore mobility with dihedral angle for the elongated and lenticular pores of a given size $R_{\mathrm{p}}=$ const are determined by Eqs. 30 and 39, respectively. These variations are considerably larger, because here the mobilities tend to infinity for $\Phi \rightarrow 0$ (wetting conditions). Also both $M_{\mathrm{p}}^{2 \mathrm{D}}(\Phi)$ and $M_{\mathrm{p}}^{3 \mathrm{D}}(\Phi)$ decrease with increasing $\Phi$ under the constraint $R_{\mathrm{p}}=$ const. For a given pore volume, however, the tendencies differ as shown in Fig. 3.

\section{Discussion}

Studying the process of pore detachment (drop) in full detail clearly requires a transient analysis. However, the few such studies that have been undertaken (Yu and Suo 1999) support the assertion that studying the existence of steady states yields reliable estimates for the critical pore velocity. Various concepts have been proposed to identify critical conditions from steady state analysis. The derived relationship between velocity and drag angle may intrinsically exhibit a maximum (Yu and Suo 1999; Petrishcheva and Renner 2005) (see also Fig. 5) for which it has been shown that it separates stable and instable solutions ( $\mathrm{Yu}$ and Suo 1999). The drag angles associated with the maximum in velocity of a lenticular pore controlled by diffusion through the pore-filling fluid (Fig. 5) increase from $\pi / 3$ to $<2 \pi / 3$ with increasing dihedral angle enclosing the value of $\pi / 2$ alternatively proposed as the condition for pore detachment from geometric reasoning (Hsueh et al. 1982; Monchoux and Rabkin 2002).

While our analysis of elongated pores is to our knowledge new, migration of lenticular pores controlled by diffusion through the pore-filling fluid has previously been addressed in classical studies on spherical pores (Shewmon 1964; Brook 1969, 1976) and in an approximate analytical approach (Monchoux and Rabkin 2002). In our notation, the classical solutions for a spherical pore read

$M_{\mathrm{p}}^{\mathrm{sph}}=\frac{2}{3} \frac{D \Omega^{2} c_{0}}{V_{\mathrm{p}}^{\mathrm{sph}} k T}$

and

$U_{\max }^{\mathrm{sph}}=\frac{1}{2} \frac{\gamma_{\mathrm{b}} D \Omega^{2} c_{0}}{R_{\mathrm{p}}^{2} k T}$

assuming that the drag force reaches its theoretical maximum at $\Theta_{p}=\pi / 2$. The relations between pore mobility and critical velocity versus dihedral angle gained in Monchoux and Rabkin (2002) (given in our notation in accord with Eq. 14)

$M_{\mathrm{p}}^{3 \mathrm{D}[\mathrm{MR}(2002)]}=M_{\mathrm{p}}^{2 \mathrm{D}[\mathrm{MR}(2002)]}=\frac{D \Omega^{2} c_{0}}{V_{\mathrm{p}} k T}$

and

$U_{\max }^{3 \mathrm{D}[\mathrm{MR}(2002)]}=\frac{4 \cos (\Phi)[1+\cos (\Phi)]}{\cos (\Phi)+\sin (\Phi)-1} \frac{\gamma_{\mathrm{b}} D \Omega^{2} c_{0}}{R_{\mathrm{p}}^{2} k T}$

were derived fixing the diffusion flux in the pore-filling fluid parallel to the pore velocity. Thus, strictly speaking the results (43) and (44) should be restricted to small dihedral angles. The differences between these previous and our current results are moderate as shown in Fig. 3 (mobilities) and in Fig. 6 (critical velocities for lenticular pores). Differences become most pronounced for fully spherical pores $(\Phi \rightarrow \pi)$ for which (Monchoux and Rabkin 2002) predicts a vanishing critical velocity in contrast to classical Eq. 42 and our results indicating that the 


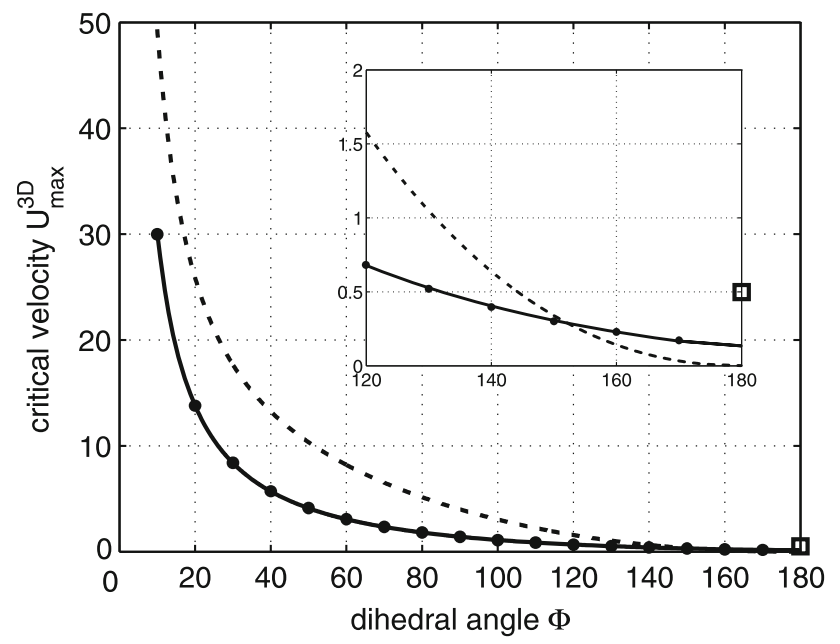

Fig. 6 Critical velocity of a lenticular pore $U_{\max }^{3 \mathrm{D}}$ normalized by $\gamma_{\mathrm{b}} D \Omega^{2} c_{0} /\left(R_{\mathrm{p}}^{2} k T\right)$ versus dihedral angle $\Phi$. The solid line represents the analytical result (38), numerical results (obtained from Fig. 5) are highlighted by points. The dashed line gives the approximate result (44) of (Monchoux and Rabkin 2002) and the open square the classic prediction for a spherical pore (42). For large dihedral angles, the relations are magnified in the inset

curvature of the flux lines cannot be neglected and the assumption of a constant critical drag angle in Monchoux and Rabkin (2002) is violated. The differences between the mobilities of lenticular pores derived here and by Monchoux and Rabkin (2002) are not large either. However, the trend with dihedral angle differs (Fig. 3).

\section{Conclusions}

We determined the dependence of mobilities and critical velocities of elongated and lenticular pores on dihedral angle $\Phi$ and pore radius $R_{\mathrm{p}}$ considering diffusive transport of matrix components through the pore-filling fluid as limiting for the pore migration. Migration is realized by a net flux of matrix constituents from the leading to the trailing pore surface. From a mathematical point of view one has to deal with the complicated solution of the diffusion problem in a permanently changing domain with the self-organized pore shape and special boundary conditions depending on the curvature of the pore-grain interfaces. We simplified this problem by approximating the leading and trailing interfaces by cylindrical or spherical segments everywhere, except for a narrow region near the pore tips. While approximate, this approach allows for the curvature of leading surfaces to switch from convex to concave with increasing velocity (see Fig. 4). This experimentally observed characteristic of pore motion was not included in the few previous approximate analyses of this problem.
Concentration fields of matrix constituents in the pores are described by Laplace equation which we solved analytically and numerically for elongated and lenticular pores, respectively. Fluxes were integrated numerically neglecting the small contribution of narrow regions near the pore tips. These regions were defined in such a way that our results correctly reproduce known values of pore mobilities for small dihedral angles which are exactly derived from the dissipation theorem following (Monchoux and Rabkin 2002).

With our analysis, we aimed to find the largest possible pore velocity for given values of $\Phi$ and $R_{\mathrm{p}}$. For elongated and lenticular pores, our results can be presented by

$U_{\max }=\frac{C_{\mathrm{u}}(\Phi)}{2 \Phi} \frac{\gamma_{\mathrm{b}} D \Omega^{2} c_{0}}{R_{\mathrm{p}}^{2} k T}$

where the second factor is uniquely determined and the first factor was estimated from the approximate solution. Analytical approximations, $C_{\mathrm{u}}^{2 \mathrm{D}}=6$ and $C_{\mathrm{u}}^{3 \mathrm{D}}(\Phi)$ [see (37)], were found for elongated and lenticular pores, respectively. At a given pore size $R_{\mathrm{p}}$, these critical velocities tend to infinity for $\Phi \rightarrow 0$ and to small but finite values for $\Phi \rightarrow \pi$. When instead considering a given volume for a fluid-filled pore, its size $R_{\mathrm{p}}$ increases with a decrease of $\Phi$ and the associated dependence of critical velocity on dihedral angle is weak.

For low pore velocities, we constrained pore mobilities of elongated and lenticular pores. At constant pore volume, the mobilities vary by less than one order of magnitude over the range of possible dihedral angles notably exhibiting increasing and decreasing values for elongated and lenticular pores with increasing dihedral angle, respectively. At constant pore size, the mobility tends to infinity for wetting conditions $(\Phi \rightarrow 0)$.

Acknowledgments This work was generously supported by the German Science Foundation (SFB 526).

Open Access This article is distributed under the terms of the Creative Commons Attribution Noncommercial License which permits any noncommercial use, distribution, and reproduction in any medium, provided the original author(s) and source are credited.

\section{Analysis of the motion of a nearly equilibrium pore}

In the following analysis, we envision the slow motion of a nearly equilibrium pore such that the pore radius $R_{\mathrm{p}}$ keeps its equilibrium value but without any further approximations for the pore shape (comp. Svoboda and Riedel 1992). The normal component of the particle flux density on the pore surface reads $j_{\mathrm{n}}=-U \cos (\varphi) / \Omega$ where $\varphi$ denotes an angle between the outward (with respect to the pore) 
normal vector $\mathbf{n}$ and pore velocity $U$. Each element $\mathrm{d} S$ of the leading surface contributes to the total particle flux $J$ in accord with

$\mathrm{d} J=-j_{\mathrm{n}} \mathrm{d} S=U \mathrm{~d} S_{\perp} / \Omega$,

where $\mathrm{d} S_{\perp}$ is obtained by projecting $\mathrm{d} S$ on a plane orthogonal to the pore velocity. The total flux reads

$J^{2 \mathrm{D}}=\frac{2 L_{\mathrm{p}} R_{\mathrm{p}} U}{\Omega}, \quad J^{3 \mathrm{D}}=\frac{\pi R_{\mathrm{p}}^{2} U}{\Omega}$

for the elongated and the lenticular pore, respectively. The renormalization procedure introduced when deriving Eq. 26 corresponds to a replacement $R_{\mathrm{p}}$ with $R_{\mathrm{p}}-\delta$. The flux $J$ is then replaced with an approximate value $\tilde{J}$ such that

$\tilde{J}^{2 \mathrm{D}}=\frac{2 L_{\mathrm{p}}\left(R_{\mathrm{p}}-\delta\right) U}{\Omega}, \quad \tilde{J}^{3 \mathrm{D}}=\frac{\pi\left(R_{\mathrm{p}}-\delta\right)^{2} U}{\Omega}$.

We see that the relative error related to the tips contribution is $\delta / R_{\mathrm{p}}$ and $2 \delta / R_{\mathrm{p}}$ for the elongated and the lenticular pore, respectively.

\section{References}

Balluffi RW, Allen SM, Carter WC (2005) Kinetics of materials. Wiley, Hoboken

Brook RJ (1969) Pore-grain boundary interactions and grain growth. J Am Ceram Soc 52:56-57

Brook RJ (1976) Controlled grain growth. In: Wang FFY (ed) Treatise on material science and technology, vol 9. Academic Press, New York, pp 331-364

Dubrovin BA, Fomenko AT (1992) Modern geometry-methods and applications. Springer, Berlin

de Groot SR (1951) Nonequilibrium thermodynamics. North Holland, Amsterdam

Haken H (2004) Synergetics: introduction and advanced topics. Springer, Berlin

Hollister L, Crawford M (eds) (1981) Fluid inclusions: applications to petrology, vol 6. Mineralogical Association of Canada, Calgary
Hsueh CH, Evans AG, Coble RL (1982) Microstructure development during final/intermediate stage sintering-I. Pore/grain boundary separation. Acta Metall 30:1269

Huang M, Wang Y, Chang YA (2004) Grain growth in sputtered nanoscale PdIn thin films. Thin Solid Films 449:113

Landau LD, Lifshitz EM (1981) Physical kinetics. Pergamon Press, USA

Lavrentjev M, Shabat B (1998) Metody teorii funkzij kompleksnogo peremennogo (in russian). Nauka, Moscow

Monchoux JP, Rabkin E (2002) Microstructure evolution and interfacial properties in the $\mathrm{Fe}-\mathrm{Pb}$ system. Acta Mater 50:3159

Mullins WW (1960) Grain boundary grooving by volume diffusion. AIME Trans 218:354

Nes E, Ryum N, Hunderi O (1985) On the zener drag. Acta Metall 33:11

Partington J (1952) An advanced treatise on physical chemistry. Longmans, London

Petrishcheva E, Renner J (2005) Two-dimensional analysis of pore drag and drop. Acta Mater 53:2793

Prigogine I (1967) Introduction to thermodynamics of irreversible processes, 3rd edn. Interscience, New York

Raj R (1982) Creep in polycrystalline aggregates by matter transport through a liquid phase. J Geophys Res 87:4731

Riedel H, Svoboda J (1993) A theoretical study of grain growth in porous solids during sintering. Acta Metall Mater 41:1929

Roedder E (1984) Fluid inclusions, vol 12. Mineralogical Society of America, Washington

Shewmon PG (1964) The movement of small inclusions in solids by a temperature gradient. Trans Metall Soc AIME 230:1134-1137

Smith CS (1948) Grains, phases, and interactions: an interpretation of microstructure. Trans Metall Soc AIME 175:15

Spears MA, Evans AG (1982) Microstructure development during final/intermediate stage sintering-II. Grain and pore coarsening. Acta Metall 30:1281

Svoboda J, Riedel H (1992) Pore-boundary interactions and evolution equations for the porosity and the grain size during sintering. Acta Metall Mater 40:2829

Thompson CV, Carel R (1996) Stress and grain growth in thin films. J Mech Phys Solids 44(5):657-673

Weaire D, Rivier N (1984) Deformation-related recrystallization processes. Contemp Phys 25:59

Yan MF, Cannon RM, Bowen HK (1977) Grain boundary migration in ceramics. In: Fulrath RM, Pask JA (eds) Ceramic microstructures. Westview Press, Boulder, pp 276-307

Yu HH, Suo Z (1999) An axisymmetric model of pore-grain boundary separation. J Mech Phys Solids 47:1131-1155 